JOURNAL OF THEORETICAL

AND APPLIED MECHANICS

54, 2, pp. 561-570, Warsaw 2016

DOI: $10.15632 /$ jtam-pl.54.2.561

\title{
PROFILE DESIGN OF NONCIRCULAR BELT PULLEYS
}

\author{
Piotr KraWieC \\ Poznań University of Technology, Chair of Basics of Machine Design, Poznań, Poland \\ e-mail:piotr.krawiec@put.poznan.pl
}

AdAM MaRlewsKI

Poznań University of Technology, Institute of Mathematics, Poznań, Poland; e-mail: adam.marlewski@put.poznan.pl

\begin{abstract}
During design of the envelope of a noncircular belt pulley one should take into account several conditions resulting from kinematics and geometry of the uneven-running strand transmission. Design of proper values of pitches for a belt pulley, which enable good cooperation between the belt pulley and the belt, should be carefully done. In available Polish literature and catalogues offered by belt manufacturers, one can find only detailed dimensions of belts with a trapezoidal profile. Information on other profiles is insufficient for full description of geometrical features of belt pulley teeth as well as to design tools for machining belt pulleys. Manufacturers of belt pulleys and belts give only necessary data to submit a purchase order. The paper presents a digitization process (scanning) of geometry of the envelope of circular belt pulleys with non-standard profiles; this scanning has been done with the application of coordinate measuring machines. The obtained information on geometrical features of tooth profiles has been numerically analysed. As a result of this analysis, the mathematical description has been obtained. Finally, noncircular belt pulleys with different tooth profiles have been designed and manufactured.
\end{abstract}

Keywords: reverse engineering, tooth profile, numerical modelling

\section{Reverse engineering: definition, historical notes and basic techniques}

Many definitions of the reverse engineering exist nowadays. In (Eilam, 2005), it is said that it is a process of extracting the knowledge or design information from anything a man made. Thus what makes the difference between the reverse engineering and conventional scientific research is that with the reverse engineering the artifact being investigated is man-made unlike in the scientific research - there is taken into account a natural phenomenon. According to Schreve and Basson (2005), the reverse engineering encompasses the set of activities aiming at (re)discovering the functional, structural and behavioural semantics of a given artifact with the aim of leveraging this information for the efficient usage or adaption of that artifact or the creation of related artifacts. In (Ülker, 2013), the reverse engineering is defined as a process of duplicating an item, i.e., its functionality and dimensions by physically examining and measuring the existing parts to develop technical data (physical and material characteristics) required for competitive procurement.

In (Eilam, 2005), the reverse engineering is dated back to the era of the industrial revolution, the transition to new manufacturing processes. Then, in the years 1760-1840, new machines entered to the manufactures, and the reverse engineering was a way of an espionage aiming at acquiring information about modern machines, to have a commercial or military advantage of having them. In fact, the espionage reverse engineering was practiced in the Antiquity: Egypt built its power on Assyrian chariots copied around 1600 BC, in the 3rd century BC Romans gained the domination on Mediterranean thanks to capturing Carthaginian quinquiremes. More 
recent examples of the analogous activities concern the Soviet piston-engine bomber Tupolev Tu-4 (it was developed in late 1940's as a copy of American Boeing B-29 aircrafts landed in Vladivostok after completing combat missions to the Japanese mainland; see e.g. Fitzpatrick and DiLullo (2006), also for juridical aspects of the reverse engineering) and decoding the Nazi German encryption machine Enigma made by Polish and British cryptographers.

All four above examples concern directly military issues. In industrial practice, the reverse engineering deals with recovering information on objects which disappeared or were damaged (during the standard use or in experiments), with creation and completing of documentation for an existing product or device, with product analysis, interoperability or re-designing.

Nowadays, the reverse engineering is applied in various branches of science and industry, and it is still the most popular in recognition of the machine element shape (e.g. Baier et al., 2012; Boyer and Petitjean, 2000; Kotlyar, 1991; Kumar, 2013; Kumar et al., 2014; Prautzsch et al., 2002; Schreve and Basson, 2005; US Army Reverse Engineering, 2006). Obviously, the reverse engineering comprises fitting, approximation and numerical optimization techniques, so it is located within the applied mathematics. Thus, an reverse engineer can not be unfamiliar with numerical methods and techniques (presented e.g. in Burden and Faires, 1985; Chapra and Canale, 1990). In industrial applications, B-splines (including NURBS and Bézier splines) are dominant representations of shape description - the reverse engineering very often applies these techniques, see. e.g. Hoschek (2001), Sarfraz et al. (2013), Weiss (2001), Xia (2014), Yin and Jiang (2010) as well as (Ray and Ray, 2013) and (Tsay and Fong, 2005) where genetic algorithms are in use. It also concerns the search for the description of non-typical gears. Such gears are discussed in many papers in both theoretical and implementation aspects - see e.g. Bär (2009), Krawiec and Marlewski (2011), Laczik (2008), Liu and Chen (2008), Liu et al. (2013), Lovasz et al. (2007), Telea (2012).

In this paper, we aim at simplifying and efficient describing the shape of a tooth which is known by a point cloud only (the coordinates of these points are obtained with the digitalization done by the CMM - coordinate measuring machine, see, e.g., Chajda et al. (2008). The tooth shape is described by circles (determined by carefully chosen points) and an appropriate parabola which joins the circles smoothly. This parabola can be presented as a Bézier arc (so it is the graph of a Bernstein polynomial; these polynomials were found in 1912 and are still investigated, see e.g. Farouki (2012), Pobegailo (2014), Prautzsch et al. (2002). This type of the description can not be produced by the existing software (such as Rhinoceros from Robert McNeel \& Associates, ZAR products from HEXAGON Software, EXCEL-LENT from Excel Gear, Inc., MITCalc Worm Gear Calculation 1.16, GearTeq from Camnetics Inc., GearEngineer from DEPO).

\section{Reverse engineering in design of belt pulleys with a noncircular envelope}

Information on geometrical features of belt pulleys with non-standard profiles can be achieved by methods of reverse engineering. In general, these methods consist of numerical discretization of elements or machines, and conversion of the obtained graphical information (points or surfaces) into two or three dimensional models (Marciniec et al., 2010). For the described example, the process of reverse engineering consisted of four stages:

- scanning of the envelope profile of a circular belt pulley with the application of the CMM,

- numerical analysis of the obtained results,

- designing of the tooth profile geometry of belt pulleys,

- manufacturing of a prototype of the noncircular belt pulley.

During the measurements, the belt pulley was mounted on the table of the CMM, and the measuring head was moved according to the assigned parameters. An essential restriction of this 
method is the possibility to scan cylindrical surfaces which are circumscribed for angles between $0^{\circ}$ and $45^{\circ}$. However, for the presented task, this range was sufficient owing to the repeatability of belt pulley profiles. As a result of the measurement, the geometrical features of the belt pulley were obtained in form of the Cartesian coordinates. The geometrical centre of the belt pulley was taken as an origin of the coordinate system. This fact has improved the design process of the geometry of noncircular belt pulleys during the next stage.

(a)

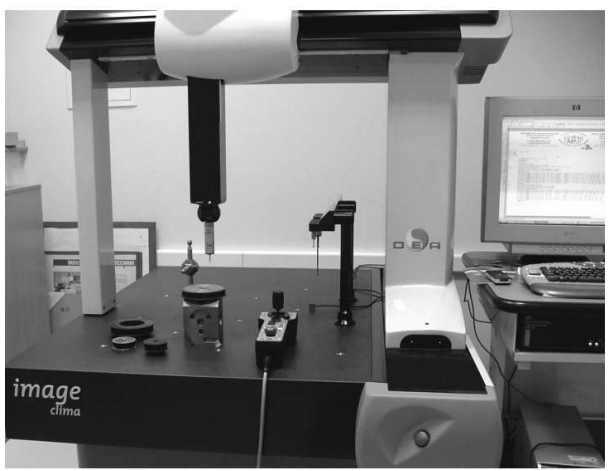

(b)

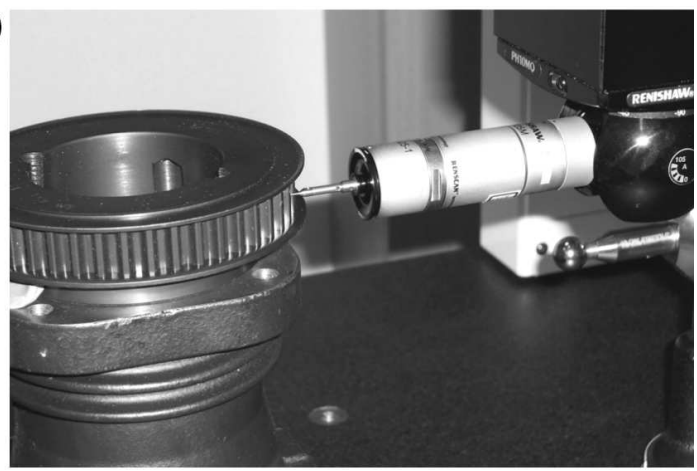

Fig. 1. Measurement of geometrical features of non-standard belt pulleys: (a) test stand, (b) belt pulley measurement

The measurement of belt pulley profiles was performed by the CMM CNC DEA Global Image Clima (Fig. 1). This machine was placed in an air-conditioned room, and this fact was essential to ensure the correctness of the measuring procedure. The measurement consisted of a continuous scanning with the use of a gauge plunger. Sampling density was equal to 100 points $/ \mathrm{mm}$, scanning speed $-5 \mathrm{~mm} / \mathrm{s}$, ball diameter of the gauge plunger SP 25M Reinshaw was equal to $1 \mathrm{~mm}$. The measurements were performed for two variants. The first one consisted of automatic application of the "offset" function - it took into account the ball dimension which was placed at the end of the gauge plunger. In application of the second variant, a fragment of the envelope profile of the belt pulley was obtained and the correction due to the ball radius was taken. The scanning process allowed one to get a set of coordinates written in a txt file. On the basis of these coordinates, the profile of the half of the belt pulley tooth was determined. The analysis of curvatures of the obtained profile allowed one to restrict the number of coordinates which are describing the profile to the value 36 (Table 1 ).

Table 1. Points coordinates accepted to the design of the tooth profile

\begin{tabular}{|c|c|c|c|c|c|c|c|c|}
\hline$j$ & $x_{j}$ & $y_{j}$ & $j$ & $x_{j}$ & $y_{j}$ & $j$ & $x_{j}$ & $y_{j}$ \\
\hline \hline 1 & -7.7752 & 55.1300 & 2 & -7.5779 & 55.1470 & 3 & -7.4664 & 55.1457 \\
\hline 4 & -7.4419 & 55.1429 & 5 & -7.3921 & 55.1449 & 6 & -7.3104 & 55.1346 \\
\hline 7 & -7.2542 & 55.1288 & 8 & -7.1365 & 55.1013 & 9 & -7.0037 & 55.0567 \\
\hline 10 & -6.9057 & 55.0085 & 11 & -6.7908 & 54.9337 & 12 & -6.7082 & 54.8588 \\
\hline 13 & -6.6734 & 54.8090 & 14 & -6.6342 & 54.7239 & 15 & -6.5970 & 54.6489 \\
\hline 16 & -6.5699 & 54.5646 & 17 & -6.5449 & 54.4684 & 18 & -6.5250 & 54.3727 \\
\hline 19 & -6.4728 & 54.1592 & 20 & -6.4334 & 53.9815 & 21 & -6.3991 & 53.8119 \\
\hline 22 & -6.3340 & 53.6141 & 23 & -6.3032 & 53.5276 & 24 & -6.2763 & 53.4198 \\
\hline 25 & -6.2224 & 53.2729 & 26 & -6.1293 & 53.1231 & 27 & -6.0630 & 53.0405 \\
\hline 28 & -6.0164 & 52.8889 & 29 & -5.8621 & 52.6834 & 30 & -5.6769 & 52.5085 \\
\hline 31 & -5.3631 & 52.2537 & 32 & -5.0184 & 52.0408 & 33 & -4.6800 & 51.8986 \\
\hline 34 & -4.1830 & 51.7606 & 35 & -3.9972 & 51.7398 & 36 & -3.7887 & 51.7423 \\
\hline
\end{tabular}


The obtained profile of the half of the tooth is presented in Fig. 2. This figure shows a square with the coordinates $\langle-8,-3\rangle \times\langle 51,56\rangle$ and the points obtained as a result of the scanning process. Five points which have a significant impact on the design process of the tooth profile are distinguished by indexes $(1,10,20,34,36)$.

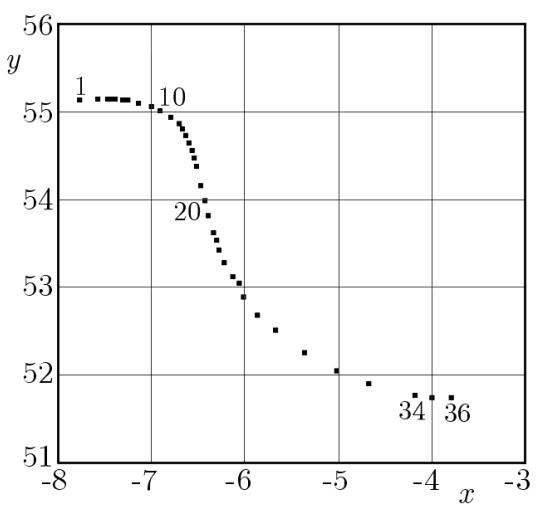

Fig. 2. Points describing the half of the tooth profile of the belt pulley

\section{Numerical determination of the tooth profile}

The points obtained during the scanning process are called measuring points, and the points which are laying on the nominal tooth profile are called nominal points.

The presented task consists in determination of mathematical description of the edge of the perfect tooth - this description should be relatively simple. According to the accepted criterion of the simplicity, the description should consist of a small number of equations and every equation should describe a "simple" curve. The simple curve is a curve which can be simply described on the basis of mathematical notation (without the application of any advanced computer programs), e.g. low degree polynomial curves (straight line, parabola and $n$-degree polynomial curves where $n$ is a small natural number - it is generally accepted that $n \leqslant 4$ ), second degree curves (circle, ellipse and hyperbola), spirals (logarithmic spiral, spiral of Archimedes) and other curves applied in mechanical engineering (e.g., cycloid, cardioid). The next research task is to find and select the so-called transition curves such as Cornu's spiral (also called as a clothoid). These curves determine a standard way of transition from a circular arc to a straight segment where the curve continuity is kept at the transition point.

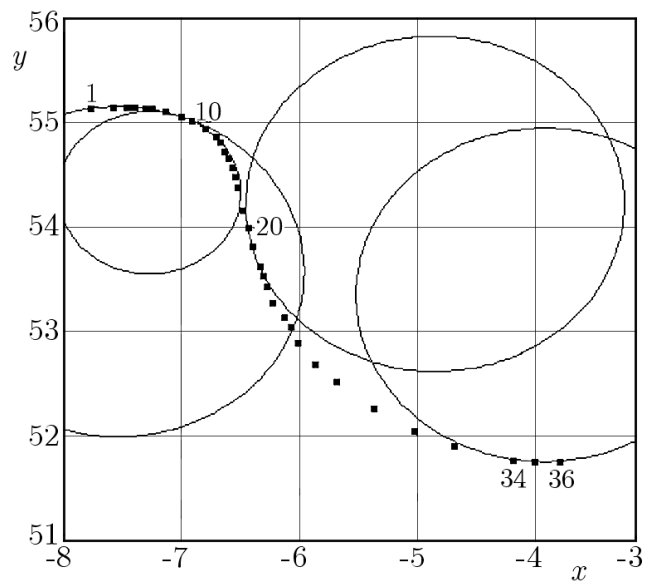

Fig. 3. Four circles which are drawn through three successive measuring points: 1-2-3, 10-11-12, 20-21-22 and 34-35-36 
Taking into account the descriptions presented in literature concerning the analysis of the measurement results of scanning (Filipowski, 2002; Filipowski et al., 2005) and information from manufacturers of belt pulleys, we decided to approximate the analysed distribution of points by arcs of tangent circles or polynomial curves of a low degree (line segments) in the case when the tangency could not be modelled. Within the framework of the preliminary analysis, the circles were drawn through every three successive measuring points. The examples of these circles are presented in Fig. 3.

On the basis of the analysis of circles position and the measuring points, we can accept that circle 10-11-12, i.e. drawn through the measuring point No. 10 and two successive points, is a good approximation of the shape in its vicinity (in the range from point No. 8 to point No. 18). Circle 34-35-36 is a satisfying approximation of the searched shape only in the vicinity of these points. Figure 4 presents nine circles which are drawn through three successive measuring points.

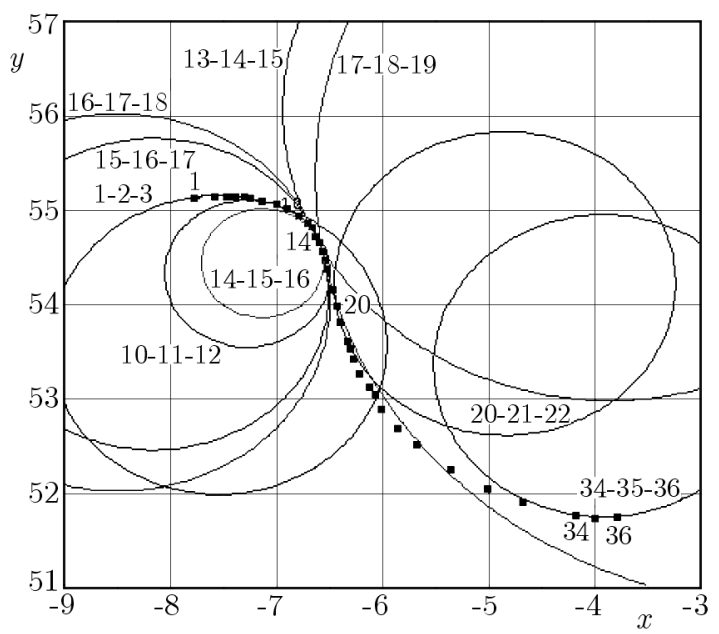

Fig. 4. Nine exemplary circles which are drawn through three successive measuring points

Radii of all 34 circles (circle No. $k$ passes through the points $k, k+1, k+2$, where $k=1,2, \ldots, 34)$ are presented in Fig. 5 . We can see that the variation of the radius $r$ has not any visible monotonicity (interval monotonicity). The interval monotonicity guarantees the fulfillment of the condition of smooth transition between elements of the tooth profile.

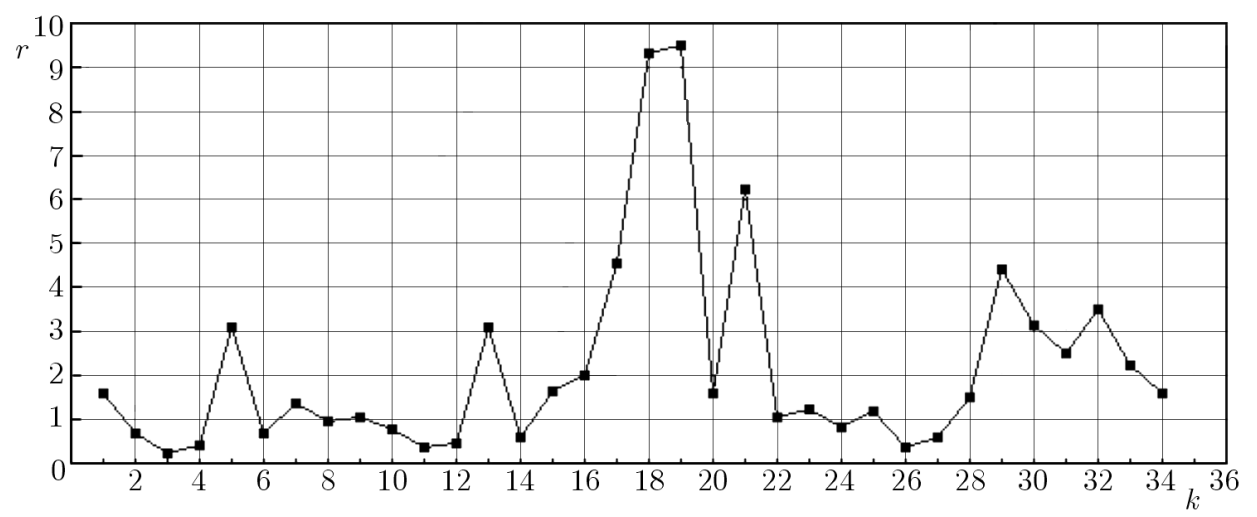

Fig. 5. Radii $r$ of circles No. $k$ for $k=1,2, \ldots, 34$. On the basis of Fig. 2, we can conclude that the radius value $r$ should be equal to 0 for point No. 16 or its vicinity; in the case of a perfect profile (when it consists of two circles) the radius values $r$ should be constant for $k$ from 1 to ca. 15 and (higher than the previous values) for $k$ from ca. 17 to 34

The interval monotonicity characterizes circles (radius $r$ is constant for any three points laying on the circle), straight lines $(r=0)$, parabolas (these curves have only one point where the 
increasing function becomes the decreasing function or vice versa), etc. For spirals, the radius $r$ is constantly increasing (or constantly decreasing), and for a standard cycloid, the radius $r$ is decreasing from the infinity first (it is decreasing to the value 0 , but it does not achieve it) and next it is increasing to the infinity. The lack of the monotonicity is not a result of the reading quality of the results from the CMM (monotonicity disturbance caused by work precision of the CMM is not high - in practice this disturbance is negligible and is a part of the so-called device error), but is a result of teeth manufacturing. Due to the lack of the monotonicity, the circles have been drawn through two nonadjacent points. An idea of this approach is visualized in Fig. 6 generated by points

$$
A=(6,7) \quad B=(7,6.82842) \quad S=(10,4.21252)
$$

The configuration of these points is intentionally similar to that exhibiting by measured 36 points (numbered from 1 to 36, see Fig. 3). They determine two circles which are tangent at the point $M$. The centres of the these circles are

$$
L=(6,4) \quad \text { and } \quad R=(10.6832,6.34164)
$$

and radii of these circles are equal to 3 and $\sqrt{5}=2.23606$, respectively (all calculations have been performed exactly with the assistance of program Derive 5 from Texas Instruments, Inc., and the results have been then rounded to six significant digits).

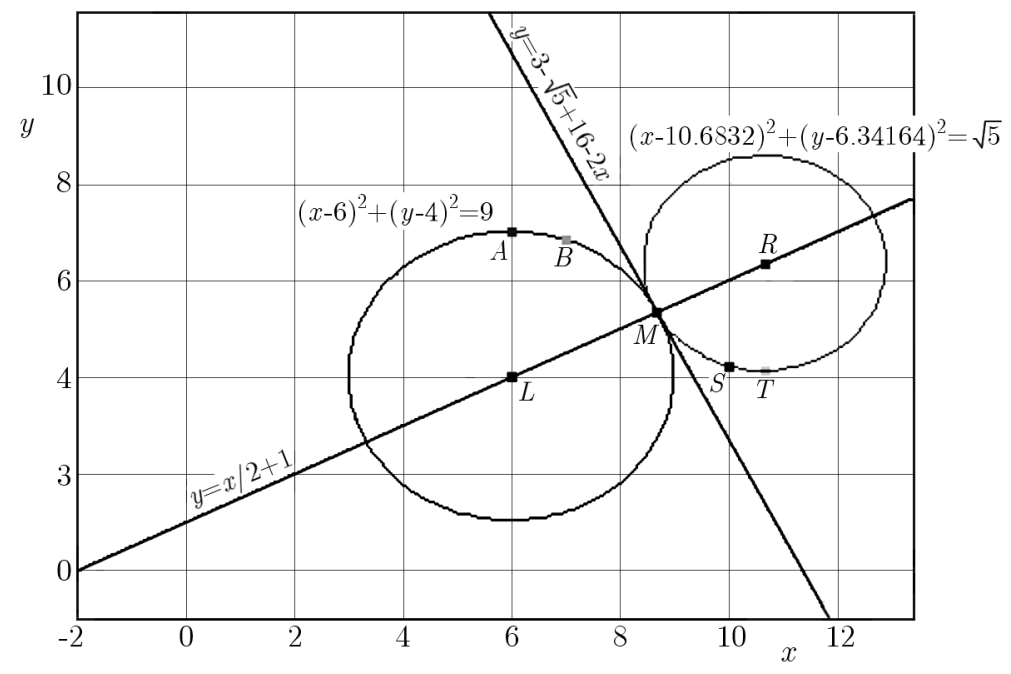

Fig. 6. Two tangent circles; the left circle is drawn through points $A$ and $B$, and the right one passes through points $S$ and $T$

The left circle has its highest point $A$, and the point $T$ is the lowest point of the right circle.

The application of this method does not allow one to find the tangent circles where one circle is drawn through the selected points from the beginning of the list (e.g. through points No. 1 and 10) and the other circle is drawn through the end points (e.g. points No. 20 and 36). The tangent circles are not found either for the case when measuring points No. 1 and 2 have not been taken into considerations. The reason of this practice is the following observation: the ordinates of these two points are smaller than the ordinates of measuring point No. 3, so (by the assumption: the reading from the CMM does not produce any significant errors) these points lay on the other side of the tooth profile than the analysed one. These points are presented in Table 1 with the measurement results, because the starting point and the end point of the reading have been imprecisely indicated.

After successive searching, it turned out that the best fitting for the end points is the circle with the lowest point No. 36, and it goes through point No. 26. The equation of this circle is

$$
(x+3.7887)^{2}+(y-54.4144)^{2}=7.14017
$$


and, as seen in Fig. 7 , on this circle the points numbered as $P_{20}, P_{21}, \ldots, P_{36}$ sit up to a high accuracy. The tangent to the circle (see Fig. 8 ) at the point $P_{20}$ has the equation

$$
y=14.6779-6.10928 x
$$

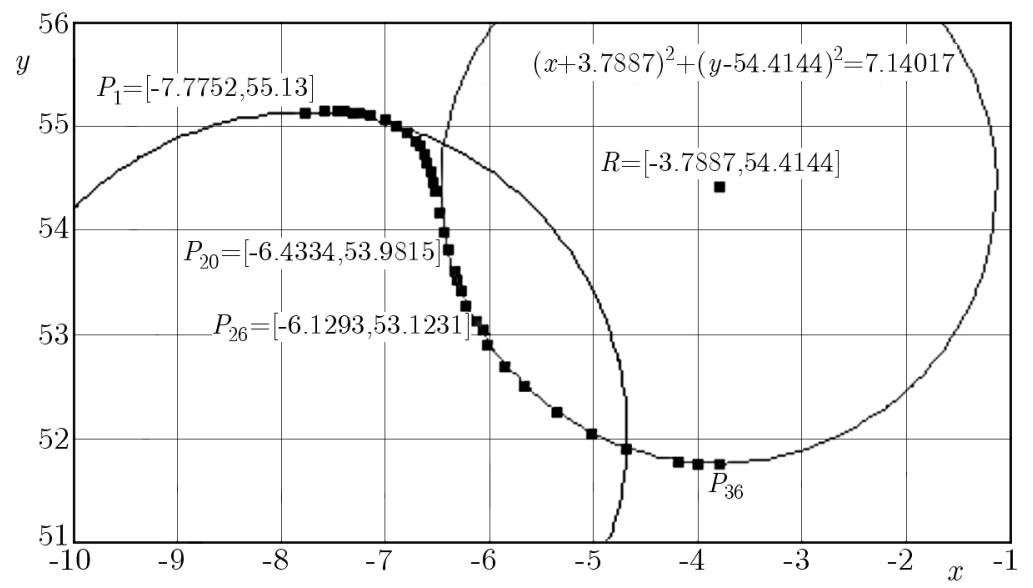

Fig. 7. Circle with the lowest point $P_{36}$ which is drawn through the point $P_{26}$

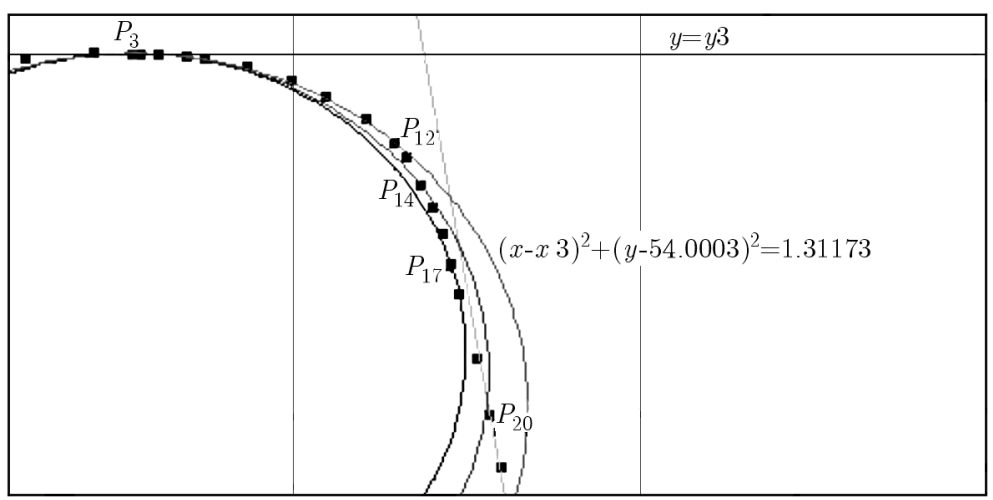

Fig. 8. Tangent line at the point $P_{20}$ and three circles for which the highest point is $P_{3}$. On the left side of the point $P_{3}$, one can see points No. 1 and 2 , and these ones are not taken into account

Searching for a circle with the highest point $P_{3}$ and a circle which passes really close to successive points is finished when the circle is drawn through the point $P_{12}$. Figure 8 shows this circle and two other circles which pass through points No. 14 and No. 17, respectively. It is clearly seen that these two other circles are worse approximations with respect to points which are close to the point $P_{3}$ than the circle which goes through the point $P_{12}$. This circle, passing through $P_{3}$ and $P_{12}$, is covered by the equation

$$
(x+7.4664)^{2}+(y-54.0003)^{2}=1.31173
$$

and the line which at $P_{12}$ is tangential to this circle has the equation

$$
y=48.9341-0.883171 x
$$

The curve between the points $P_{12}$ and $P_{20}$ is found by an inverse search for the Bernstein polynomial $B_{2}$ of degree 2 . Here, we know points the wanted polynomial passes through (the points $P_{12}$ and $P_{20}$ ) and slopes of tangent lines at these points (respectively -0.883171 and $-6.10928)$, so we can find coefficients $c_{0}, c_{1}$ and $c_{2}$ of the polynomial

$$
x \rightarrow B_{2}(t):=c_{0} t^{2}+2 c_{1} t(1-t)+c_{2}(1-t)^{2}
$$


where the variable

$$
t=3.63901 x+24.4112
$$

is introduced in order to transform the interval $\left\langle x_{12}, x_{20}\right\rangle=\langle-6.7082,-6.4334\rangle$ in the standard interval $\langle 0,1\rangle$ which is used to define the Bernstein polynomial $B_{2}$. It results with the equation

$$
y=-7.18927 x^{2}-97.6709 x-276.821
$$

It describes the parabola which joins smoothly (i.e. with continuous derivatives at points $P_{12}$ and $\left.P_{20}\right)$ the $\operatorname{arc} P_{3} P_{12}$ of the left circle and the $\operatorname{arc} P_{20} P_{36}$ of the right circle.

As we see, the application the Bernstein interpolation (let us call so what we did: we joined two circular arcs by a parabola arc fitted in form of the Bernstein polynomial) resulted with a polynomial curve (of the lowest possible degree here, 2). This is a non typical use of the Bernstein polynomials (by their nature they serve to approximate points, and not to interpolate them). A standard procedure to get a curve connecting two other curves is to deal with an Hermite interpolating polynomial or with a spline function; in our case they have to be found for the curvilinear segment with ends in $P_{12}$ and $P_{20}$, and we obtain a polynomial of the third degree. Thus, our non-typical Bernstein interpolation produced a polynomial of a lower degree (2); it means we obtained a description we looked for, namely the one of degree 2 only. It is important because the lower degree the less sensitivity to data, and the data are measured quantities (so they are always approximate values) as well as to the roundings executed in both the calculation process and the final form (here it embraces 6 significant digits). Therefore, the arc of the polynomial curve of the third degree satisfies the imposed requirements worse than the polynomial curve $y=B_{2}(x)$ - especially in relation to the value $y_{12}$. The obtained approximation satisfies (even in excess) the limits of manufacturing tolerances for the belt pulley teeth.

To verify the correctness of the conducted scanning process, numerical data processing and CAD modelling, a few of sets of noncircular belt pulleys were manufactured. Due to the low manufacturing costs and relatively high manufacturing accuracy, the FDM (fused deposition modelling) method of rapid prototyping was applied (Fig. 9).

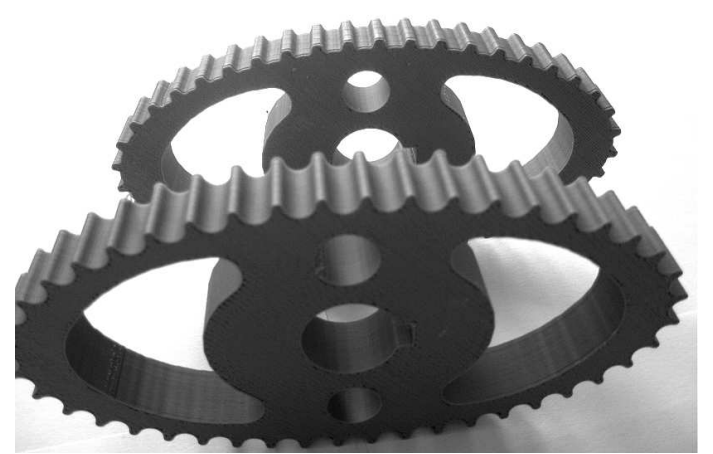

Fig. 9. Noncircular belt pulleys with nonstandard tooth profiles manufactured with the use of the rapid prototyping method

The manufactured belt pulleys were subjected to metrological analysis in the scope of geometrical features. The assessment of the correctness of the coupling between the belt and the belt pulleys was also done with the test stand. The results of these analyses show that the presented methodology can be applied with success to determine tooth profiles which are not defined in standards. 


\section{Summary}

The reverse engineering presented in this paper starts with a cloud of points laying in the plane, the coordinates of these points are obtained by the CMM (coordinate measuring machine), and aims at producing a simple mathematical description of the object points. In the reported case, the digitalized points are taken from a recently produced belt pulley which has not any geometrical specification. The obtained description consists of three equations: for the arcs of two circles and the parabola which smoothly joins them. This description is worked out via examination of several other ones, and the paper shows how the final result has been obtained. This method serves as an exemplary approach to the reverse engineering which can be applied in analogous situations when, due to the definition of the shape, standard procedures are not applicable or these ones give formulas which do not fulfil the requirement for the simplicity. A positive result of the discussed procedure confirms that the reverse engineering can be successfully used to retrieve, as well as to design, the teeth geometry of non-typical gears. It also shows the specificity of finding of an appropriate mathematical description; this process is inventory (it has to be carefully fitted to the point cloud at hand) and time-consuming, it is troublesome to execute it with no computer assistance. It also suggests that it is worthy to undertake research on the automatization of forming of the mathematical description of profiles, and such a description is necessary for numerical control of producing machines.

\section{References}

1. Baier M., Bogucki R., Baier A., 2012, Reverse engineering as a tool in process of gear reconstruction, Selected Engineering Problems, 3, 9-14

2. B ̈̈r G.F., 2009, Two synthesis methods for non-circular cylindrical gears, Journal of Geometry and Graphics, 13, 1, 101-112

3. Boyer E., Petitjean S., 2000, Curve and surface reconstruction from regular and non-regular point sets, Proceedings of Conference on Computer Vision and Pattern Recognition, Hilton Head Island, 2, 659-665

4. Burden R.L., FAIRes J.D., 1985, Numerical Analysis, PWS Publishers, Boston

5. Chapra S.C., Canale R.P., 1990, Numerical Methods for Engineers, McGraw Hill International Editions

6. Chajda J., ET AL., 2008, Coordinate measurement of complicated parameters like roundness, cylindricity, gear teeth or free-form surface, 8 Intermational Conference of Advanced Manufacturing Operations Kranevo, 225-231

7. Eilam E., 2005, Reversing - Secrets of Reverse Engineering, John Wiley \& Sons

8. Farouki R.T., 2012, The Bernstein polynomial basis: a centennial retrospective, Computer Aided Geometric Design, 29, 379-419

9. Fitzpatrick W.M., DiLullo S.A., 2006, Attack of the clones: reverse engineering, R\&D and the law, Competition Forum, 4, 2, 501-515

10. Filipowski R., 2002, Generating and Processing of Offset Curves and Machine Element Surfaces with the Application of CAD/CAM (in Polish), Warsaw, Publishing House of Poznan University of Technology

11. Filipowski R., Dąbrowski L., Zawora J., 2005, New method of offset points generation for scanning with CMM (in Polish), Pomiary Automatyka Kontrola, 11, 5-8

12. HoscheK J., 2001, Mathematical methods in reverse engineering, [In:] Maultivariate Approximation and Applications, N. Dyn, D. Leviatan, D. Levin, A. Pinkus (Edit.), Cambridge University Press, 252-284 
13. Kotlyar Y., 1991, Gear fundamentals reverse engineering, Gear Technology, 7, 34-38

14. Krawiec P., Marlewski A., 2011, Spline description of not typical gears for belt transmissions, Journal of Theoretical and Applied Mechanics, 49, 2, 355-367

15. Kumar B.R., 2013, Polygonal approximation and scale-space analysis of clcosed digital curves, Apple Academic Press, 388

16. Kumar A., Jain P.K., PathaK P.M., 2014, Curve reconstruction of digitized surface using K-means algorithm, Procedia Engineering, 69, 544-549

17. LACZIK B., 2008, Design and Manufacturing of Non-circular Gears by Given Transfer Function, HEXAGON Industriesoftware GmbH http://www.hexagon.de/pdf/noncgear.pdf; also Laczik B. - Analytic Design of Non-circular Gears by Maple, http://www.maplesoft.com/ applications $/$ view.aspx?SID $=6885 \&$ view $=$ html

18. Liu G., Chang K., Liu Z., 2013, Reverse engineering of machine-tool settings with modified roll for spiral bevel pinons, Chinese Journal of Mechanical Engineering, 26, 3, 573-584

19. Liu J., Chen Y., 2008, A design for the pitch curve of noncircular gears with function generation, International MultiConference of Engineers and Computer Scientists, II, 1681-1686

20. Lovasz E.C., Peruu D., Modler K.-H., Margineanu D.T., Zabava E.S., 2007, Non-circular gear wheels in the geared-linkages mechanisms, 12th IFToMM World Congress, Besançon, paper A394, p. 6

21. Marciniec A., Borowiak M., Dziubek K., 2010, Reconstruction of gear geometry with application of CMM and CAD system (in Polish), Pomiary Automatyka Kontrola, 56, 1, 60-61

22. Pobegailo A.P., 2014, Interpolating Bézier spline curves with local control, Buletinul Academiei de Stiinte a Republicii Moldova Moldova, Matematica, 75, 2, 18-28

23. Prautzsch H., Boehm W., Paluszny M., 2002, Bézier- and B-spline Techniques, Springer-Verlag, 304

24. RAY K.S., RAY B.K., 2013, Polygonal approximation of digital curve based on reverse engineering concept, Internation Journal of Image and Graphics, 13, 1350017

25. Sarfraz M., Irshad M., Hussain M.Z., 2013, Reverse engineering of planar objects using GAs, Sains Malaysiana, 48, 2, 1167-79

26. Schreve K., Basson A.H., 2005, Edge detection in reverse engineering using a scanning approach. Part I: Scanning algorithm, International Journal of Advanced Manufacturing Technology, 26, 1048-1054

27. TeleA A.C., 2012, Reversing. Reverse Engineering. Recent Advances and Applications, In Tech

28. Tsay M., Fong Z., 2005, Study on the generalized mathematical model of noncircular gears, Mathematical and Computer Modelling, 41, 555-569

29. ÜLKer E., 2013, B-spline curve approximation using Pareto envelope-based selection algorithm PESA, International Journal of Computer and Communication Engineering, 2, 1, 60-63

30. US Army Reverse Engineering, 2006, Military Handbook MIL-HDBK-115A

31. Weiss V., 2001, Reverse engineering free-form shapes, PhD dissertation presented at University of Technology and Economics, Budapest, 106

32. XIA Z., 2014, Application of reverse engineering based on computer in product design, International Journal of Multimedia and Ubiquitous Enginering, 9, 5, 343-354

33. Yin Z., Jiang S., 2010, Automatic segmentation and approximation of digitized data for reverse engineering, International Journal of Production Research, 41, 13, 3045-58 